\title{
MODELING TEMPORAL DEPENDENCE OF SPHERICALLY INVARIANT RANDOM VECTORS WITH TRIPLET MARKOV CHAINS
}

\author{
Nicolas Brunel and Wojciech Pieczynski \\ GET/INT, CITI Department, UMR-CNRS 5157 \\ 9, rue Charles Fourier, 91011 Evry Cedex, France
}

\begin{abstract}
Our paper deals with multivariate hidden Markov chains (MHMC) with a view towards segmentation. We propose a new model in which temporal dependencies are modelled using copulas and sensor dependencies are represented by Spherically Invariant Random Vector (SIRV). Copulas are very useful and flexible tools, which have been little applied in signal processing problems until now. In particular, for some desirable marginal distributions it is possible to obtain different kind of dependencies. Using some recent results on Triplet Markov chains, the new model extends the case of MHMC when the observations are SIRV and independent conditionally on the states. We propose algorithms for computing efficiently the posterior probabilities of the involved Triplet Markov Chain, in order to propose rapid segmentation and estimation procedures.
\end{abstract}

\section{INTRODUCTION}

Spherically Invariant Random Vectors are important in different radar signal or image modeling and processing. In fact, such a model enables one to deal with non Gaussian and correlated multivariate distributions, which are very useful in numerous situations $[9,19]$.

Let $Y=\left(Y^{1}, \ldots, Y^{M}\right)$ be a random vector taking its values in $R^{M}$. The vector $Y$ is called Spherically Invariant Random Vector (SIRV) if there exists a random Gaussian vector $V=\left(V^{1}, \ldots, V^{M}\right)$ called "speckle", and a positive real random variable $\tau$ called "texture", such that $Y=\sqrt{\tau} V$. This representation enables also to introduce various statistical laws for modeling intensities in radar images as in [7]. We will use the following "inverse" representation (already used in [8]) which is better fitted for computational purpose: putting $U=\tau^{-1}$, we have $Y=U^{-1 / 2} V$. We will call the distributions of $Y^{1}, \ldots, Y^{M}$ "marginal distributions" of $Y$, or simply "margins" of $Y$. For some distributions of $U$, the margins are calculable; for example, if $U$ has a gamma distribution, the margins are Student laws, and when $U$ has an inverse gamma distribution, the margins are K laws. In SIRV, the margins define the whole distribution of $Y$, which is somewhat restricting. One possible way to remedy this restriction is to use copulas in which it is possible to consider different joint distributions with fixed marginal distributions [14].

More precisely, let $Z_{1}, \ldots, Z_{M}$ be $M$ real random variables, with $F_{1}, \ldots, F_{M}$ their respective cumulative distribution functions (cdf), and $F$ the cdf of the law of $Z=\left(Z_{1}, \ldots, Z_{M}\right)$. If $F_{1}, \ldots, F_{M}$ are continuous, which will be assumed in this paper, then according to Sklar's theorem there exists an unique function $C:[0,1]^{M} \longrightarrow[0,1]$, called "copula", such that $F\left(z_{1}, \ldots, z_{M}\right)=C\left(F_{1}\left(z_{1}\right), \ldots, F_{M}\left(z_{M}\right)\right) \quad$ for every $\left(z_{1}, \ldots, z_{M}\right) \in R^{M}$. An important property is that for a random vector $Z=\left(Z_{1}, \ldots, Z_{M}\right)$ the associated copula models the dependence of its components $Z_{1}, \ldots, Z_{M}$ in an intrinsic way, independently of the margins. More precisely, let $Z^{*}=\left(Z_{1}^{*}, \ldots, Z_{M}^{*}\right)$ be another random vector defined from $Z=\left(Z_{1}, \ldots, Z_{M}\right)$ by $Z_{i}^{*}=\varphi_{i}^{*}\left(Z_{i}\right), i=1 . . M$ where the $\varphi_{i}^{*}$ 's are non-decreasing functions from $R$ to $R$. Then $Z$ and $Z^{*}$ have the same copula, while the margins vary according to $\varphi_{1}^{*}, \ldots, \varphi_{M}^{*}$. In particular, let $Z$ be a random vector, $C$ the associated copula, and let us consider $M$ distributions on $R$ given by the cdf $F_{1}^{*}, \ldots, F_{M}^{*}$. Then considering $Z_{1}^{*}=\varphi_{1}^{*}\left(Z_{1}\right), \ldots$, $Z_{M}^{*}=\varphi_{M}^{*}\left(Z_{M}\right)$ with $\varphi_{i}^{*}\left(z_{i}\right)=\left(F_{i}^{*}\right)^{-1} \circ F_{i}\left(z_{i}\right), i=1 . . M$, we obtain $Z^{*}=\left(Z_{1}^{*}, \ldots, Z_{M}^{*}\right)$ such that the associated copula is $C$ and the margins are $F_{1}^{*}, \ldots, F_{M}^{*}$. Thus we see that for a given copula we can obtain any desired distribution for the components of $Z^{*}$. Another important property, especially for simulation, is that $C$ is the cdf of the standardized vector $\left(F_{1}\left(Z_{1}\right), \ldots, F_{M}\left(Z_{M}\right)\right)$. The cross- 
derivative function $c=\frac{\partial^{M}}{\partial u_{1} \cdots \partial u_{M}} C\left(u_{1}, \cdots, u_{M}\right)$ is then called the density of the copula.

Let us assume that there is a sequence of SIRV $Y_{1}=\left(Y_{1}^{1}, \ldots, Y_{1}^{M}\right), \ldots, \quad Y_{N}=\left(Y_{N}^{1}, \ldots, Y_{N}^{M}\right), \quad$ such as the distribution of each $Y_{n}=\left(Y_{n}^{1}, \ldots, Y_{n}^{M}\right)$ depends on a hidden state $X_{n}=x_{n}$ in $\Omega=\left\{\omega_{1}, \ldots, \omega_{K}\right\}$. Multivariate Hidden Markov Chain (MHMC) is then a classical model enabling one to deal with such situations. In the classical MHMC the sequence $X=\left(X_{1}, \ldots, X_{M}\right)$ is a Markov chain, and the random variables $Y_{1}, \ldots, Y_{N}$ are independent conditionally on $X$, and such as $p\left(y_{n} \mid x\right)=p\left(y_{n} \mid x_{n}\right)$ (the conditional density of $Y_{n}$ on $X$ is equal to its conditional distribution on $X_{n}$ ) for each $n=1, \ldots, N$. Such a model can then be used in different problems; in this paper, we will focus on estimation of the hidden states $X=\left(X_{1}, \ldots, X_{N}\right)$ from the observed multivariate sequence $Y=\left(Y_{1}, \ldots, Y_{N}\right)$, that is the classical problem in image and signal processing of the Bayesian segmentation. Our aim is to specify a conditional process $\left(Y_{n} \mid X_{1}^{N}\right)_{n \geq 1}$ with SIRV margins which is not necessarily a sequence of independent variables.

Very recently, copulas have been introduced in hidden Markov chains in two kinds of situations:

(i) for $M=1$, one can use copulas to introduce temporal dependencies of $Y_{1}, \ldots, \quad Y_{N}$ conditionally on $X=\left(X_{1}, \ldots, X_{N}\right)$ [3], which can be made either in the classical context of HMC, or in the more general context of "Pairwise" Markov chains [18];

(ii) for any $M$, one can use copulas to model the dependence among sensors, in the classical context of independence of $Y_{1}, \ldots, Y_{N}$ conditionally on $X$ as in the previous paragraph [2].

We will simultaneously consider (i) and (ii) by adding dependencies in the texture chain $U=\left(U_{1}, \ldots, U_{N}\right)$ so that the three chains $X, U, Y$ can be modeled as a "Triplet Markov chain" (TMC [12,17]). In the next section, we will describe in detail the TMC model proposed, and we will emphasize some interesting properties. Then, we deal with the essential problem of the computation of the posterior probabilities and we propose a Sequential Monte Carlo algorithm for filtering and smoothing. Finally, we address the problem of estimation of the TMC, in order to perform unsupervised segmentation.

\section{MODEL AND SOME PROPERTIES}

Let us consider the SIRV model in the classical MHMC context above. Thus we have two sequences
$X=\left(X_{1}, \ldots, X_{M}\right)$ and $Y=\left(Y_{1}, \ldots, Y_{M}\right)$; according to the very definition of SIRV there is also a third chain $U=\left(U_{1}, \ldots, U_{M}\right)$ such as the density can be written

$p\left(y_{n} \mid x_{n}\right)=\int_{0}^{+\infty} p\left(y_{n} \mid x_{n}, u_{n}\right) p\left(u_{n} \mid x_{n}\right) d u_{n}$

Therefore, we can say that there are three random processes $\quad X=\left(X_{1}, \ldots, X_{N}\right), \quad U=\left(U_{1}, \ldots, U_{N}\right), \quad$ and $Y=\left(Y_{1}, \ldots, Y_{N}\right)$ verifying:

(i) $X$ is a Markov chain

(ii) $\left(U_{1}, Y_{1}\right), \ldots,\left(U_{N}, Y_{N}\right)$ are independent conditionally on $X$ and such that $p\left(u_{n}, y_{n} \mid x\right)=p\left(u_{n}, y_{n} \mid x_{n}\right)$ for each $n=1, \ldots, N$.

We see that the triplet $T=(X, U, Y)$ is a Markov chain, and thus the model can be seen as a particular "triplet" Markov chain. Our aim is to extend this model to a more general one, in which the possibility of estimating $X$ from $Y$ would remain.

Let the hidden couple $(X, U)$ be a Markov chain, with transition kernel having density

$$
p\left(x_{n+1}, u_{n+1} \mid x_{n}, u_{n}\right)=p\left(x_{n+1} \mid x_{n}\right) p\left(u_{n+1} \mid x_{n+1}, x_{n}, u_{n}\right)
$$

We still assume that the observations $Y_{1}, \ldots, Y_{N}$ are independent conditionally on $(U, X)$ and that $p\left(y_{n} \mid u, x\right)=p\left(y_{n} \mid u_{n}, x_{n}\right)$ for each $n=1, \ldots, N$. We stress on the fact that $Y_{1}, \ldots, Y_{N}$ are no more independent conditionally on $X$. In other words, by introducing some dependence (conditionally on $X$ ) among the texture variables $U_{1}, \ldots, U_{N}$, we introduce some dependence among $Y_{1}, \ldots, Y_{N}$ (conditionally on $X$ ), and we get a more general model.

The dependence between the $U_{i}$ 's is modeled, as in [3], by a copula. Thus following [3], with the chain $U$ instead of $Y$, we can define a "hidden Markov chain with copulas" $(X, U)$. The density of the transition kernel of the chain $(X, U)$ is:

$$
\begin{aligned}
p\left(x_{n+1}, u_{n+1} \mid x_{n}, u_{n}\right)= & p\left(x_{n+1} \mid x_{n}\right) g\left(u_{n+1} \mid x_{n+1}\right) \\
& \times c\left(G\left(u_{n} \mid x_{n}\right), G\left(u_{n+1} \mid x_{n+1}\right)\right)
\end{aligned}
$$

where $g$ is the probability density function (pdf) of $U_{n}$ conditionally on $X_{n}$ (and $G$ the corresponding cdf). By the use of copulas, we can choose the density $g$ independently of the dependence structure, so we can always have the desired expression for $p\left(y_{n} \mid x_{n}\right)$. For example, if the margins of $U$ are gamma distributed (conditionally on $X$ ), then we get a process $Y$ having Student margins (conditionally on $X$ ). 
Remark 2.1: The introduction of a copula in order to have a correlated Markovian texture has a proper interest for designing models of stationary processes with SIRV margins. If there is one class $\left(\Omega=\left\{\omega_{1}\right\}\right), Y$ is a classical Hidden Markov Chain, with a continuous hidden state $U$. Some examples of stationary process with SIRV margins have been introduced for modeling correlated $\mathrm{K}$ clutter in [13] in coherent radar detection context, but our model is parsimonious since the dependence structure of the observations is completely described by the Markovian dependence (represented by the copula $C$ ) of the hidden state.

The model can also be seen as an extension of some stochastic volatility (SV) models, the latter being used in econometrics to model the volatility of asset returns. Some SV models are HMC, having the following statespace representation

$\left\{\begin{array}{cl}U_{n+1}=\rho U_{n}+\sigma \varepsilon_{1, n} & \varepsilon_{1, n} \sim N(0,1) \\ Y_{n}=\beta \exp \left(U_{n} / 2\right) \varepsilon_{2, n} & \varepsilon_{2, n} \sim N(0,1)\end{array}\right.$

$Y_{n}$ is the observed process of asset prices, and $\beta \exp \left(U_{n} / 2\right)$ is the volatility, so $U_{n}$ is termed the logvolatility instead of texture. The randomness of the volatility enables then to reproduce the heavy tails observed on real data, such as Student laws. Nevertheless, the dynamic of $U_{n}$ is often linear (autoregressive process) in order to be able to identify the stationary behavior of the volatility $U$, and hence of the observed process.

The use of copulas for describing the dynamic of $U_{n}$ enables to consider any non-linear first order dependency and any stationary law for $U_{n}$ and for $Y_{n}$, among the family of SIRV (see the relationship between texture and clutter laws in [19]). For instance, if we use a Gaussian copula with density

$$
c(u, v ; \rho)=\left(1-\rho^{2}\right)^{-1 / 2} \exp \left(-\frac{\varsigma_{1}^{2}+\varsigma_{2}^{2}-2 \rho \varsigma_{1} \varsigma_{2}}{2\left(1-\rho^{2}\right)}+\frac{\varsigma_{1}^{2}+\varsigma_{2}^{2}}{2}\right)
$$

and $\zeta_{1}=\Phi^{-1}(u), \varsigma_{2}=\Phi^{-1}(v), \Phi^{-1}$ being the inverse of the standardized Gaussian cdf, the state space representation of the process $Y$ conditionally on $X$ (by removing the states $\left.X_{n}\right)$ is:

$$
\left\{\begin{array}{cc}
\Phi^{-1}\left(G\left(U_{n+1}\right)\right)=\rho \Phi^{-1}\left(G\left(U_{n}\right)\right)+\sqrt{1-\rho^{2}} \varepsilon_{1, n} & \varepsilon_{1, n} \sim N(0,1) \\
Y=\sigma U^{-1 / 2} \varepsilon & \varepsilon \sim N(0,1)
\end{array}\right.
$$

The equation of evolution of the "volatility" is not linear, and it suffices to adapt $G$ in order to have directly the desired law for a better fitting of the model to the data. The proposed model has three main properties:

(i) the law of $Y$ conditionally on $X$ is neither independent nor Markovian.

(ii) the above dependence is controlled by copulas, so that we can describe various and numerous kinds of dependence (elliptical or Archimedean copulas among others) $[11,14]$.

(iii) the margins of $Y$ are controlled and belongs to parametric families which have been proved to be adequate for describing real data (in Radar, Image Processing, or Finance and Econometrics), and exhibiting heavy tails.

Finally the model can also deal with complex observations (usual in radar) by considering complex SIRV via a complex Gaussian vector $V$. Moreover, in order to deal with a general problem of segmentation, we can also consider that the mean level is discriminating, hence we introduce a shift in the mean: $Y_{n}=m+U_{n}^{-1 / 2} V_{n}$. The obtained law still belongs to the family of elliptical laws [10].

\section{SMOOTHING AND SEGMENTATION}

We present in this section the effective computation of the posterior densities $p\left(x_{n} \mid y_{1}^{N}\right)$ by using the celebrated forward-backward formulas of HMM, adapted to the context of TMC [18]. Nevertheless, the computational weight is increased because of the necessity of the computation of the joint posterior density $p\left(x_{n}, u_{n} \mid y_{1}^{N}\right)$, and we propose a Sequential Monte Carlo (SMC) algorithm to deal with the composite (discrete and continuous) nature of the hidden process. In order to limit the computational burden and to avoid the simulation of discrete particles, we use the algorithm proposed independently by Perez and Vermaak [16] in a mathematically equivalent model. Contrary to us, Perez et $a l$. want to retrieve the continuous process $U$ from the observations, but the same equations apply. Hence, we present first a SMC algorithm for the stationary HMC $(U, Y)\left(\Omega=\left\{\omega_{1}\right\}\right)$ and derive the final algorithm for the TMC.

\subsection{Smoothing for HMC $(U, Y)$}

The process is indexed by the mean vector $m \in \mathbb{R}^{M}$ and the variance matrix $\Sigma \in \mathbb{R}^{M \times M}$ of the Gaussian conditional vector $Y_{n} \mid U_{n}$ (whose density is noted $f(y \mid u)$ dropping $m$ and $\Sigma$ ). The stationary density of the Markov chain is indexed by a parameter $g=g_{\theta}$, as the copula $C=C_{\alpha}$, with density $c=c_{\alpha}$.

\subsubsection{Filtering}

We propose here a regularized particle filter, called postregularized by Musso, Oudjane and Le Gland [15], based on a Gaussian kernel with periodic resampling. We simulate at each iteration $n$ a population of $D$ particles 
$\left(\zeta_{i}^{n}\right)_{1 \leq i \leq D}$, with corresponding weights $\left(w_{i}^{n}\right)_{1 \leq i \leq D}$. The regularized particle approximation of the forward density $p\left(u_{n} \mid y_{1}^{n}\right)$ is

$\pi_{n}^{(r)}(u)=\sum_{i=1}^{D} w_{i}^{n} \phi_{h}\left(u-\zeta_{i}^{n}\right)$

where $\phi_{h}$ is the Gaussian centered density with standard deviation $h, h$ being the optimal bandwidth of the nonparametric estimator of the density with the Gaussian kernel. The cdf of $\pi_{n}^{(r)}$ is $\Pi_{n}^{(r)}(u)=\sum_{i} w_{i}^{n} \Phi\left(\left(u-\zeta_{i}^{n}\right) / h\right)$. At $n=1$, the particles $\left(\zeta_{i}^{1}\right)_{1 \leq i \leq D}$ are drawn according to $g_{\theta}$ and have weights $w_{i}^{1}=f\left(y_{1} \mid \zeta_{i}^{1}\right) / \sum_{j} f\left(y_{1} \mid \zeta_{j}^{1}\right)$.

For $n \geq 1$, the prediction step is:

- draw $D$ particles $\left(\zeta_{(i)}^{n}\right)_{1 \leq i \leq D}$ from the density $\tilde{\pi}_{n}$. Then simulate $\forall i \leq D, \varpi_{i}^{n} \in[0,1]$ such as the joint cdf of $\left(\Pi_{n}^{(r)}\left(\varsigma_{(i)}^{n}\right), \varpi_{i}^{n}\right)$ is the copula $C_{\alpha}$. The propagated particle is $\zeta_{i}^{n+1 \mid n}=G_{\theta}^{-1}\left(\varpi_{i}^{n}\right)$, so that the joint cdf of $\left(\varsigma_{(i)}^{n}, \varsigma_{i}^{n+1 \mid n}\right)$ is $C\left(\Pi_{n}^{(r)}, G_{\theta}\right)$. The predictive density $\pi_{n+\mid n}(u)=p\left(u_{n+1} \mid y_{1}^{n}\right)$ can be approximated by $\sum_{i=1}^{D} w_{i}^{n} p\left(u_{n+1} \mid \zeta_{i}^{n}\right)$.

And the correction step is:

- Update the weights by

$$
w_{i}^{n+1} \propto w_{i}^{n} \times f\left(y_{n+1} \mid \zeta_{i}^{n+1}\right) \times \frac{c_{\alpha}\left(G_{\theta}\left(\zeta_{(i)}^{n}\right), G_{\theta}\left(\zeta_{i}^{n+1}\right)\right)}{c_{\alpha}\left(\Pi_{n}^{(r)}\left(\zeta_{(i)}^{n}\right), G_{\theta}\left(\zeta_{i}^{n+1}\right)\right)}
$$

since the instrumental transition density is $q_{n}\left(u \mid u^{\prime}\right)=g_{\theta}(u) c_{\alpha}\left(\Pi_{n}^{(r)}\left(u^{\prime}\right), G_{\theta}(u)\right)$.

The use of this instrumental kernel enables to put emphasis on the influence of the copula on the dependence between the particles and between the observations. Moreover, the true and the instrumental transition kernels share the same dependence structure (copula) and one margin, consequently the instrumental distribution is expected to be close to the true distribution.

\subsubsection{Backward procedure and smoothing}

Thanks to our knowledge of the stationary distribution of $U_{n}$, we can propose a SMC algorithm for computing the backward procedure, in order to obtain easily a particle approximation of the marginal posterior density. For stationary Markov chains, the backward procedure can be interpreted as the forward procedure of the time reversed process $(\tilde{U}, \tilde{Y})=\left(U_{N-n+1}, Y_{N-n+1}\right)_{1 \leq n \leq N} \quad$ [6]. Indeed, the backward density can be rewritten

$$
\begin{aligned}
\beta_{n}\left(u_{n}\right) & =p\left(y_{n+1}^{N} \mid u_{n}\right)=\frac{p\left(\tilde{y}_{1}^{N-n}, \tilde{u}_{N-n+1}\right)}{P\left(\tilde{u}_{N-n+1}\right)} \\
& \propto \frac{p\left(\tilde{u}_{N-n+1} \mid \tilde{y}_{1}^{N-n}\right)}{g_{\theta}\left(\tilde{u}_{N-n+1}\right)}
\end{aligned}
$$

The joint process $(\tilde{U}, \tilde{Y})$ is still a HMC, and $\tilde{U}$ has the following transition density:

$p\left(\tilde{u}_{n+1} \mid \tilde{u}_{n}\right)=g_{\theta}\left(\tilde{u}_{n+1}\right) c_{\alpha}\left(G_{\theta}\left(\tilde{u}_{n+1}\right), G_{\theta}\left(\tilde{u}_{n}\right)\right)$

so we can use a similar SMC algorithm in order to compute the particle approximation of the predictive densities of the process $\tilde{U}$. The simulated particles are then sorted in the non-reverse order so that $\left(\tilde{\zeta}_{i}^{n}\right)_{1 \leq i \leq D}$ with weights $\left(\tilde{w}_{i}^{n}\right)_{1 \leq i \leq D}$ approximate the predictive densities by $\tilde{\pi}_{N-n+\| N-n}(u)=\sum_{j=1}^{D} \tilde{p}\left(u \mid \tilde{\zeta}_{j}^{n+1}\right) \tilde{w}_{j}^{n+1} . \quad$ Using $\quad$ Eq.(3.4), the deduced approximation of the backward density is

$\beta_{n}(u) \propto \sum_{j=1}^{D} c_{\alpha}\left(G_{\theta}(u), G_{\theta}\left(\tilde{\zeta}_{j}^{n+1}\right)\right) \tilde{w}_{j}^{n+1}$

Hence the smoothing densities are approximated by:

$$
p\left(u_{n} \mid y_{1}^{N}\right)=\sum_{i, j=1}^{D} w_{i}^{n} c_{\alpha}\left(G_{\theta}\left(\varsigma_{i}^{n}\right), G_{\theta}\left(\tilde{\zeta}_{j}^{n+1}\right)\right) \tilde{w}_{j}^{n+1} \delta_{\varsigma_{i}^{n}}(u)
$$

\subsection{Smoothing for composite TMC $(X, U, Y)$}

The tracking algorithm of Perez et al. enables to keep the previous simple smoothing algorithm, and to make a direct computation of the probabilities $p\left(x_{n} \mid y_{1}^{N}\right)$, without the need of a joint simulation of $\left(X_{n}, U_{n}\right)$. As shown in Eq. (2.2), the transition density is the product of the transition densities of $X$ and $U$. The latter is noted $p\left(u_{n+1} \mid u_{n}, x_{n+1}, x_{n}\right)=p_{x_{n}, x_{n+1}}\left(u_{n+1} \mid u_{n}\right)$. The transitions of the discrete process is given by the matrix $A=\left(a_{i j}\right)$, and we suppose that $A$ has a unique positive stationary distribution $v$, represented by the vector $\left(v_{i}\right)_{1 \leq i \leq M} \in \mathbb{R}^{M}$. We modify the notation of the density of the Gaussian conditional vector to take into account the dependence toward $X_{n}: f_{x_{n}}\left(y_{n} \mid u_{n}\right)$ that depends on the discrete and the continuous part of the process. The forward density can then be factorized $p\left(x_{n}, u_{n} \mid y_{1}^{n}\right)=p_{x_{n}}\left(u_{n} \mid y_{1}^{n}\right) p\left(x_{n} \mid y_{1}^{n}\right)$, so that the conditional forward density $p\left(u_{n} \mid y_{1}^{n}, x_{n}\right)=p_{x_{n}}\left(u_{n} \mid y_{1}^{n}\right)$ appears. Finally, for the segmentation of the process $Y$, we are interested in the marginal probability $\xi_{x_{n}}^{n}=p\left(x_{n} \mid y_{1}^{n}\right)$ of the joint forward probability. 


\subsubsection{Filtering for composite TMC}

It is possible to update recursively the two parts of the joint filtering probability thanks to a set a successive recurrences.

The first step is the computation of $p_{x_{n}}\left(u_{n} \mid y_{1}^{n}\right)=\frac{p_{x_{n}}\left(u_{n}, y_{n} \mid y_{1}^{n-1}\right)}{p_{x_{n}}\left(y_{n} \mid y_{1}^{n-1}\right)} \quad$ (subscript $\quad x_{n} \quad$ indicates conditioning on $X_{n}=x_{n}$ ) whose numerator can be decomposed

$$
\begin{aligned}
p_{x_{n}}\left(u_{n}, y_{n} \mid y_{1}^{n-1}\right)= & \sum_{x_{n-1}} p_{x_{n-1}, x_{n}}\left(u_{n}, y_{n} \mid y_{1}^{n-1}\right) \\
& \times p\left(x_{n-1} \mid x_{n}, y_{1}^{n-1}\right)
\end{aligned}
$$

with

$p_{x_{n-1}, x_{n}}\left(u_{n}, y_{n} \mid y_{1}^{n-1}\right)=f_{x_{n}}\left(y_{n} \mid u_{n}\right) \int p_{x_{n-1}, x_{n}}\left(u_{n} \mid u_{n-1}\right) p_{x_{n-1}}\left(u_{n-1} \mid y_{1}^{n-1}\right) d u_{n-1}$ and $\tilde{a}_{x_{n-1}, x_{n}}^{n}=p\left(x_{n-1} \mid x_{n}, y_{1}^{n-1}\right) \propto a_{x_{n-1}, x_{n}} \xi_{i}^{n}$. The denominator, termed conditional predictive likelihood, is obtained by integrating Eq.(3.6)

$$
p_{x_{n}}\left(y_{n} \mid y_{1}^{n-1}\right)=\sum_{x_{n-1}} \int p_{x_{n-1}, x_{n}}\left(u_{n}, y_{n} \mid y_{1}^{n-1}\right) p\left(x_{n-1} \mid x_{n}, y_{1}^{n-1}\right) d u_{n}
$$

The final recursions are

$$
\begin{aligned}
& p_{x_{n}}\left(u_{n} \mid y_{1}^{n}\right)=\frac{\sum_{x_{n-1}} \tilde{a}_{x_{n-1}, x_{n}}^{n} p_{x_{n-1}, x_{n}}\left(u_{n}, y_{n} \mid y_{1}^{n-1}\right)}{p_{x_{n}}\left(y_{n} \mid y_{1}^{n-1}\right)} \\
& \xi_{x_{n}}^{n}=\frac{p_{x_{n}}\left(y_{n} \mid y_{1}^{n-1}\right) \sum_{x_{n-1}} a_{x_{n-1}, x_{n}} \xi_{x_{n-1}}^{n-1}}{\sum_{x_{n}} p_{x_{n}}\left(y_{n} \mid y_{1}^{n-1}\right) \sum_{x_{n-1}} a_{x_{n-1}, x_{n}} \xi_{x_{n-1}}^{n-1}}
\end{aligned}
$$

As in [16], if we use a SMC algorithm to compute the probabilities $p_{x_{n}}\left(u_{n} \mid y_{1}^{n}\right)$, we can adapt the simulation of the population of particles by using proposal transition density of the form $q_{n}\left(u_{n} \mid u_{n-1}, x_{n}, x_{n-1}\right)$ corresponding to the joint distribution with cdf $C_{\alpha}\left(G_{\theta}\left(u_{n} \mid x_{n}\right), G_{\theta}\left(u_{n+1} \mid x_{n+1}\right)\right)$.

\subsubsection{Backward procedure and smoothing}

We can still use a forward procedure to achieve the backward procedure, and the smoothing step. We introduce the time-reversed process $\tilde{X}=\left(X_{N-n+1}\right)_{1 \leq n \leq N}$, so that we rewrite Eq. (3.3) by adding the discrete process:

$\beta_{n}\left(u_{n}, x_{n}\right) \propto \frac{P\left(\tilde{u}_{N-n+1}, \tilde{x}_{N-n+1} \mid \tilde{y}_{1}^{N-n}\right)}{P\left(\tilde{u}_{N-n+1}, \tilde{x}_{N-n+1}\right)}$

The density of the stationary law of the hidden process $(X, U)$ is equal to the product $v_{x_{n+1}} g_{\theta}\left(u_{n+1} \mid x_{n+1}\right)$, and we only need to extend the recursions to the predictive filter density

$$
p\left(u_{n}, x_{n} \mid y_{1}^{n-1}\right)=\sum_{x_{n-1}} \int a_{x_{n-1}, x_{n}} p_{x_{n-1}, x_{n}}\left(u_{n} \mid u_{n-1}\right) p\left(u_{n-1}, x_{n-1} \mid y_{1}^{n-1}\right) d u_{n-1}
$$
whose computation is direct.
The particle approximation of the continuous part of the forward density gives also a particle approximation of the backward density and of the marginal posterior density $p\left(u_{n}, x_{n} \mid y_{1}^{N}\right)$. For segmentation, we need only to sum over the particles to obtain an approximation of the probability $P\left(x_{n} \mid y_{1}^{N}\right)$.

\section{ESTIMATION}

Inference in Hidden Markov Models (HMM) is often done by the Maximum Likelihood Estimator (MLE) because of the existence of consistency results in a broad variety of HMM, and also because of the existence of numerous algorithms (amongst them the celebrated EM algorithm) to compute it [4].

The cornerstone of the success of these iterative methods (EM, SEM, ICE) is the ability to compute rapidly and recursively the posterior probabilities of the hidden process $\left(U_{n}\right)$ or $\left(X_{n}, U_{n}\right)$. For instance, if we consider the estimation of the HMC $(U, Y)$ (when $\Omega=\left\{\omega_{1}\right\}$ ), the EM algorithm is based on the "complete log-likelihood"

$$
\begin{aligned}
\log \left(p\left(u_{1}^{N}, y_{1}^{N}\right)\right) & =-\frac{N}{2} \log \left((2 \pi)^{M}|\Sigma|\right)+\sum_{n=1}^{N} \frac{M}{2} \log \left(u_{n}\right)-u_{n} p_{n} \\
& +\sum_{n=1}^{N} \log \left(g_{\theta}\left(u_{n}\right)\right)+\sum_{n=1}^{N-1} \log \left(c_{\alpha}\left(G_{\theta}\left(u_{n}\right), G_{\theta}\left(u_{n+1}\right)\right)\right)
\end{aligned}
$$

with $p_{n}$ the quadratic form of the Gaussian density.

The "E step" involves only the computation of the posterior densities $p\left(u_{n} \mid y_{1}^{N}\right)$ and $p\left(u_{n}, u_{n+1} \mid y_{1}^{N}\right)$, which can be done thanks to the recursions described in the previous section. Finally, iterative estimation of the parameters can also be performed by the method proposed by Cappé in [5] due to the additive form of the projected $\log$-likelihood $E\left[\log \left(p\left(U_{1}^{n}, y_{1}^{n}\right)\right) \mid y_{1}^{n}\right]$.

\section{CONCLUSIONS}

We have presented in this paper a new model for the segmentation of SIRV within the setting of Triplet Markov Models which enables to remove the usual assumption of conditional independence. We stress on the fact that the introduction of a correlated hidden scalar process is relevant for modeling real phenomenon since it corresponds to common and validated models of stationary process in the Radar and Econometric communities for representing radar pulses or volatility returns. Moreover, the original use of copulas in the modeling of the dependence of a hidden process can be applied to the construction of HMC where we need to control the shapes of the laws of the observations. This gives an additional degree of freedom for use on real data. Despite the increase of complexity in the model and the 
presence of a continuous hidden process, we have show that there still exists recursive, efficient and simple procedures for the computation of posterior probabilities, and hence for segmentation and estimation. We focus here on the estimation of the state process, but exactly the same machinery can be used for the estimation of the texture process, as in [16] and it may be an interesting continuation to perform and compare the estimation of the continuous and the discrete process on real radar data. An additional development is the study and comparison of different estimators of the TMC amongst the different existing techniques for HMC [4].

\section{REFERENCES}

[1] R. Barakat. Direct derivation of intensity and phase statistics of speckle produced by a weak scatterer from the random sinusoid model. J. Opt. Soc. Amer., 71(1), pp. 86-90, 1981.

[2] N. Brunel, W. Pieczynski and S. Derrode, Copulas in vectorial hidden Markov chains for multicomponent image segmentation, ICASSP'05, Philadelphia, USA, March 2005.

[3] N. Brunel and W. Pieczynski, Unsupervised signal restoration using hidden Markov chains with copulas, Signal Processing, 2005 (to be published).

[4] O. Cappé, E. Moulines and T. Ryden, Inference in Hidden Markov Models, Springer-Verlag, 2005.

[5] O. Cappé, Recursive computation of smoothed functionals of hidden Markovian processes using a particle approximation, Monte Carlo and applications, 7(1-2), pp. 81-92, 2001.

[6] Y. Chen and T.L. Lai, Sequential Monte Carlo Methods in filtering and smoothing in Hidden Markov Models, Technical Report, Department of Statistics, Stanford University, 2003.

[7] Y. Delignon and W. Pieczynski. Modeling non-Rayleigh speckle distribution in SAR images, IEEE Trans. on Geoscience and Remote Sensing, 40(6) :1430-1435, 2002.

[8] F. Gini, A. Farina, Vector subspace detection in compoundgaussian clutter. Part I: Survey and new results, IEEE Trans. On Aerospace and Electronic systems, Vol. 38, No. 4, pp. 12951311, October 2002.

[9] F. Gini and M. Greco. Covariance matrix estimation for CFAR detection in correlated heavy tailed clutter. Signal Processing, 82(12), pp. 1847-1859, December 2002.

[10] A.K. Gupta and T. Varga, Elliptically contoured models in statistics, Mathematics and its applications, Kluwer Academic Publ., 1993.

[11] H. Joe. Multivariate Models and Dependence Concepts, volume 73 of Monographs on Statistics and Applied Probability. Chapman and Hall, 1997.
[12] P. Lanchantin and W. Pieczynski, Unsupervised non stationary image segmentation using triplet Markov chains, Advanced Concepts for Intelligent Vision Systems (ACIVS 04), Aug. 31-Sept. 3, Brussels, Belgium, 2004.

[13] P. Lombardo, A. Farina, Coherent radar detection against K-distributed clutter with partially correlated texture, Signal Processing, 48, pp. 1-15, 1996.

[14] R. B. Nelsen. An introduction to Copulas. Number 139 in Lecture notes in Statistics. Springer-Verlag, 1998.

[15] C. Musso, N. Oudjane and F. LeGland, Improving Regularized Particle Filter, in Sequential Monte Carlo Methods in Practice, A. Doucet, N. de Freitas and N. Gordon Eds. Statistics for engineering and information science, Springer, 2001.

[16] P. Perez and J. Vermaak, Visual Tracking and auxiliary discrete processes, International Symposium on Applied Stochastic Models and Data Analysis (ASMDA), May 17-20 2005.

[17]. W. Pieczynski, C. Hulard, and T. Veit, Triplet Markov Chains in hidden signal restoration, SPIE's International Symposium on Remote Sensing, September 22-27, Crete, Greece, 2002.

[18] W. Pieczynski, Pairwise Markov chains, IEEE Trans. on Pattern Analysis and Machine Intelligence, Vol. 25, No. 5, pp. 634-639, 2003.

[19] M. Rangaswamy, D. Weiner, and A. Ozturk. Non-Gaussian random vector identification using spherically invariant random process. IEEE Trans. on Aerospace and Electronic Systems, 29(1), pp. 111-124, January 1993. 